\title{
Femtosecond Laser Tomography Using the TriBeam System
}

\author{
T.M. Pollock ${ }^{1}$, M.P. Echlin ${ }^{1}$, A. Mottura ${ }^{2}$
}
${ }^{1}$ Materials Department, University California at Santa Barbara, Building 503 - Materials
Department, Santa Barbara, CA, USA, 93106-5050

${ }^{2}$ School of Metallurgy and Materials, University of Birmingham, Birmingham, UK

The recently developed TriBeam system [1] combines a dualbeam FIB system with a femtosecond laser for the controlled ablation of material and 3D tomography. The TriBeam can be used for ultrafast laser machining inside an electron microscope for the removal of thin layers of material, ranging from roughly $50 \mathrm{~nm}$ to $>10 \mu \mathrm{m}$ per slice. The newly exposed laser ablated surface is imaged using a combination of SE, BSE, ISE, EBSD, and EDS for topological, chemical, and structural material information. This technique has been used to create 3D datasets and reconstruction for a wide variety of materials including metallic alloys, composites, and ceramics. EBSD data has been directly collected from laser-machined surfaces of most of these materials.

A strontium titanate (STO) dataset was collected and reconstructed in order to investigate grain growth models and develop TriBeam datasets complementary to X-ray tomography datasets. A micropillar of STO was investigated using diffraction contrast tomography (DCT) at a series of heat treatments [2] and has been sectioned with the TriBeam to define the locations of grain boundaries. The gathered dataset will be discussed as well as the merger of the data from the DCT experiments with the TriBeam dataset.

Polycrystalline nickel-base alloy René 88DT was serial sectioned using the TriBeam system to gather grain cluster information in 3D. Two datasets were gathered, one in the volume surrounding a fatigue initiation site from a high-cycle fatigue experiment, and another larger volume from a bulk sample. The two datasets were analyzed using a criteria defined by Miao et al [3], which predicts the location of highest probability fatigue crack initiation sites based on: grain size, relative grain orientation, Schmid factor, and the presence of a $\Sigma 3$ twin boundary. We have applied these conditions to the dataset in order to determine the size of the volume statistically required to contain an initiation site.

Datasets have also been gathered from a thermoelectric NiTiSn alloy, which contains Heusler and half-Heusler phases. Both of these phases are cubic with very similar d-spacing, making them difficult to index using EBSD. The EBSD master patterns have been simulated for the microscope conditions used in this experiment by M. DeGraef (CMU), and clearly show the dominant [220] reflections for both half and full Heusler phases. Phase identification has been made using both EDS assisted indexing and by weighting the indexation to reflectors that are unique to each phase. The reconstructed dataset gives insight into the phase connectivity, which may give rise to the distinct change in conductivity seen in these alloys with increasing Ni content. Grain orientation information also shows the location of grain boundaries, which are likely to act as phonon scattering sites. 
[1] MP Echlin et al. Review of Scientific Instruments 83 (2012) p. 023701.

[2] M Syha et al. Scripta Materialia 66 (2012) p.1-4 .

[3] J Miao et al. Acta Materialia 60 (2012) p.2840-2854. 